\title{
¿Más horas en la escuela incrementan el logro escolar? Evidencia para el nivel secundaria del Programa de Escuelas de Tiempo Completo en México
}

\section{Does Spending Longer at School Increase Educational Attainment? Evidence from the Full-Time School Program in Junior High Schools in Mexico}

\author{
Alma Sofía Santillán Hérnández (*) https://orcid.org/0000-0001-8736-8366
}

(*) Universidad Autónoma del Estado de Hidalgo

(Recibido: 31 de octubre de 2019; Aceptado para su publicación: 30 de marzo de 2020)

Cómo citar: Santillán, A. S. (2021). ¿Más horas en la escuela incrementan el logro escolar? Evidencia para el nivel secundaria del programa de escuelas de tiempo completo en México. Revista Electrónica de Investigación Educativa, 23, e24, 1-15. https://doi.org/10.24320/redie.2021.23.e24.3540

\section{Resumen}

El objetivo de este artículo es presentar evidencia sobre el impacto del Programa de Escuelas de Tiempo Completo de México en el porcentaje de estudiantes de tercer grado de secundaria que son clasificados en los niveles de logro insuficiente y sobresaliente. El impacto se estima utilizando el método de emparejamiento por puntajes de propensión en las escuelas incorporadas por primera vez al programa en el ciclo escolar 2014-2015. El impacto se estima para los años 2015, 2017 y 2019. Los resultados muestran que no hay efecto significativo del programa en el logro escolar en Matemáticas.

Palabras clave: política educativa, escuelas de tiempo completo, rendimiento escolar, escuela secundaria

\begin{abstract}
This article aims to offer evidence on the impact of Mexico's Full-Time School Program (PETC) on the percentage of third-grade junior high school students displaying unsatisfactory or outstanding levels of attainment. This impact was estimated using propensity score matching for schools joining the program for the first time in the 2014-2015 school year. The impact is estimated for 2015, 2017 and 2019. The results show no significant effect from the program on attainment in mathematics; this null effect persists 4 years after the program started.
\end{abstract}

Keywords: educational policy, full-time school, academic achievement, junior high school

\section{Introducción}

La educación es un medio para que los individuos cuenten con mejores condiciones laborales, mayores ingresos y condiciones de vida más igualitarias (Abdullah et al., 2015; Card, 1999). Además, en la literatura especializada se muestra que una mayor calidad de la educación conduce en el largo plazo a tener empleos con mayores ingresos (Hanushek y Wößmann, 2007). ${ }^{1}$ En esta dirección, las personas que habitan en

\footnotetext{
${ }^{1}$ Los autores consideran educación de calidad aquella que incrementa las habilidades de los individuos.
} 
localidades marginadas se ven limitadas para acceder en el futuro a mejores condiciones de vida, en virtud de que tienen menores oportunidades de recibir una educación de calidad (Secretaría de Educación Pública [SEP], 2017).

En México, el debate sobre la calidad educativa ha estado presente en diversos escenarios. Desde lo político se han tratado de implementar mecanismos que lleven a los niños a recibir una educación con mayor calidad. No obstante, estos mecanismos han sido criticados porque no hay claridad en los métodos que deberán ser utilizados para mejorar la calidad de la educación (López-Aguilar, 2013).

De acuerdo con el modelo de Carroll (1963), el aprendizaje depende directamente del tiempo destinado al estudio, e inversamente al tiempo necesario para aprender, por lo que al aumentar el tiempo de clases se espera que se alcance el aprendizaje. En una modificación del modelo, la teoría del dominio de aprendizaje de Bloom (1973) observa que las desigualdades individuales en el aprendizaje son reflejo de los distintos tiempos necesarios para aprender, de tal manera que es posible alcanzar niveles parecidos de aprendizaje entre poblaciones con diversas habilidades por medio de una asignación heterogénea del tiempo de estudio, es decir, proporcionando más tiempo de clase a aquellas personas con resultados deficientes. Siguiendo los conceptos plasmados en estos modelos, el tiempo de estudio representa las horas de clases.

De manera empírica, uno de los medios reconocidos en el mundo por tener efectos positivos en la educación es el incremento en el número de horas de clase (Bellei, 2009; Cattaneo et al., 2017; Meroni y Abbiati, 2016; Rivkin y Schiman, 2015). Por ejemplo, en China, uno de los tres países con los mejores resultados en la prueba PISA de 2015 (OCDE, 2016a), se dedican entre 8 y 9.5 horas a impartir clases. Por otro lado, de acuerdo con los resultados de esta prueba en México, donde en las escuelas públicas del nivel secundaria las horas de instrucción escolar son 6, el 57\% de los jóvenes no alcanza el nivel básico de competencias en matemáticas (OCDE, 2016b).

De acuerdo con lo estipulado en el artículo 3o. de la Constitución de los Estados Unidos Mexicanos: "El Estado garantizará la calidad de la educación de manera que los materiales y métodos educativos, la organización escolar, la infraestructura educativa y la idoneidad de los docentes y directivos garanticen el máximo aprendizaje en los estudiantes" (Art. 3o. Reformado, 2013). En 2001 los ministros de educación de Latinoamérica y el Caribe, en la Declaración de Cochabamba (2001), expresaron su preocupación sobre la calidad educativa y establecieron la recomendación de ampliar el horario de clases al menos a 1000 horas y el ciclo escolar a 200 días de clases. Con base en esta recomendación y con la finalidad de mejorar la calidad educativa en el nivel básico, en 2007 el Gobierno Federal implementó el Programa de Escuelas de Tiempo Completo (PETC), que consiste en incrementar entre 2 y 4 horas la estancia en secundaria.

Existen análisis previos de los efectos del PETC sobre el rendimiento escolar (Andrade-Baena, 2014; Cabrera-Hernández, 2015; Santillán, 2014; Silveyra et al., 2018). En su mayoría, estos estudios utilizan sólo los resultados de la Evaluación Nacional de Logro Académico en Centros Escolares (ENLACE), empleando información disponible hasta el 2016. En general, se han encontrado efectos positivos y significativos del PETC sobre el rendimiento escolar en primaria, pero no hay análisis previos sobre sus efectos en secundaria.

Este artículo aporta elementos para la discusión sobre la estimación causal del incremento en el número de horas de clase sobre el rendimiento escolar en secundaria. Para ello, se usaron datos de escuelas telesecundarias beneficiarias del PETC y los resultados de la prueba del Plan Nacional para la Evaluación de los Aprendizajes (Planea) aplicada en 2015, 2017 y 2019. Planea es un examen estandarizado reciente que no cuenta con los problemas de medición que presentaba la ENLACE, la cual dejó de aplicarse por su baja confiabilidad (Instituto Nacional para la Evaluación de la Educación [INEE], 2018). Por lo tanto, al emplearse una prueba que ha sido mejorada y blindada, los resultados son más acertados para medir el logro escolar.

Para medir el efecto tratamiento del programa se utiliza el método de emparejamiento por puntajes de propensión. Se calcula el efecto para las escuelas telesecundarias que participaron por primera vez del PETC en el ciclo escolar 2014-2015. Como grupo de comparación se consideran dos muestras: la primera formada por telesecundarias que no fueron beneficiarias del programa en el ciclo escolar 2014-2015; y la segunda por telesecundarias que tampoco ingresaron en ese ciclo escolar, pero que entraron al PETC en 
los ciclos 2015-2016, 2016-2017, 2017-2018 o 2018-2019.

Los resultados de este estudio muestran que en Matemáticas no hay efecto significativo en el porcentaje de estudiantes con nivel de logro insuficiente, ni en el nivel de logro sobresaliente. En el caso de Lenguaje tampoco se observa efecto significativo en el logro escolar con el grupo de comparación de las telesecundarias que entraron al programa después del ciclo escolar 2014-2015.

\subsection{Descripción del Programa}

El PETC consiste principalmente en incrementar las jornadas escolares entre 2 y 4 horas diarias. El número de horas adicionales de clase se determina con base en las condiciones y necesidades locales. El objetivo de este incremento es que se fortalezca el aprendizaje de las asignaturas establecidas en el plan de estudios, de una lengua adicional, del arte y la cultura. Con fines de equidad, en escuelas ubicadas en zonas pobres y marginadas, el PETC impulsa esquemas para proporcionar alimento a los estudiantes.

De acuerdo con las reglas de operación para el ciclo escolar 2014-2015 (Acuerdo número 704 por el que se emiten las Reglas de Operación del Programa Escuelas de Tiempo Completo, 2013), el programa está dirigido a escuelas primarias y telesecundarias de un solo turno. Además, las escuelas deben de cumplir al menos uno de los siguientes criterios: atender una población en situación vulnerable o en contexto de rezago social, tener bajos niveles de logro educativo o altos índices de deserción y estar ubicadas en zonas en las que opera la Cruzada contra el Hambre ${ }^{2}$ y el Programa Nacional para la Prevención Social de la Violencia y la Delincuencia. ${ }^{3}$

La decisión de participar en el programa es voluntaria. Primero, cada entidad federativa interesada firma un convenio en el que acepta participar. Posteriormente, las escuelas se postulan ante las autoridades educativas estatales para ser beneficiarias y realizan una propuesta local para implementar y desarrollar el programa. La selección final de las escuelas receptoras se basa en la estrategia local para el desarrollo de la educación y en la disponibilidad presupuestal. Las escuelas beneficiarias reciben asesoría para mejorar las capacidades de gestión, para favorecer la participación de la comunidad escolar y para mejorar los resultados académicos en términos de rendimiento escolar, aprobación y retención.

Adicionalmente, el PETC otorga subsidios a las escuelas beneficiarias en una única exhibición durante el ciclo escolar; hasta $61 \%$ del recurso otorgado puede utilizarse para el pago de apoyo económico a directivos, docentes y personal de apoyo del programa. El resto del dinero puede ser empleado en el fortalecimiento de la autonomía de gestión en la escuela, el pago del servicio de alimentación y en la implementación local del programa. El porcentaje del recurso que puede ser destinado en cada rubro depende de los criterios establecidos en las reglas de operación.

El PETC inició en el ciclo escolar 2007-2008 en 499 escuelas del nivel básico de 15 entidades federativas de México. En secundarias el programa inició operaciones en 13 escuelas ubicadas en dos entidades. Para el ciclo escolar 2018-2019 se contaron 25631 escuelas de nivel básico pertenecientes a este programa, equivalente al $11 \%$ de las instituciones educativas del país, beneficiando a más de 3.5 millones de estudiantes de los 32 estados.

Entre 2012 y 2014 el PETC aumentó considerablemente su cobertura debido a modificaciones presupuestales. En particular, en el ciclo escolar 2014-2015 el número de telesecundarias dentro del programa aumentó 277\%; es por la magnitud de este aumento que se realiza el análisis con el grupo de telesecundarias que fueron beneficiarias por primera vez en este ciclo escolar (ver figura 1).

\footnotetext{
2 Estrategia del Gobierno Federal que busca reducir el hambre de las personas en pobreza extrema.

3 Programa orientado al diseño de acciones para atender los factores de riesgo vinculados a la violencia y a la delincuencia.
} 
Figura 1. Evolución del número de escuelas pertenecientes al PETC

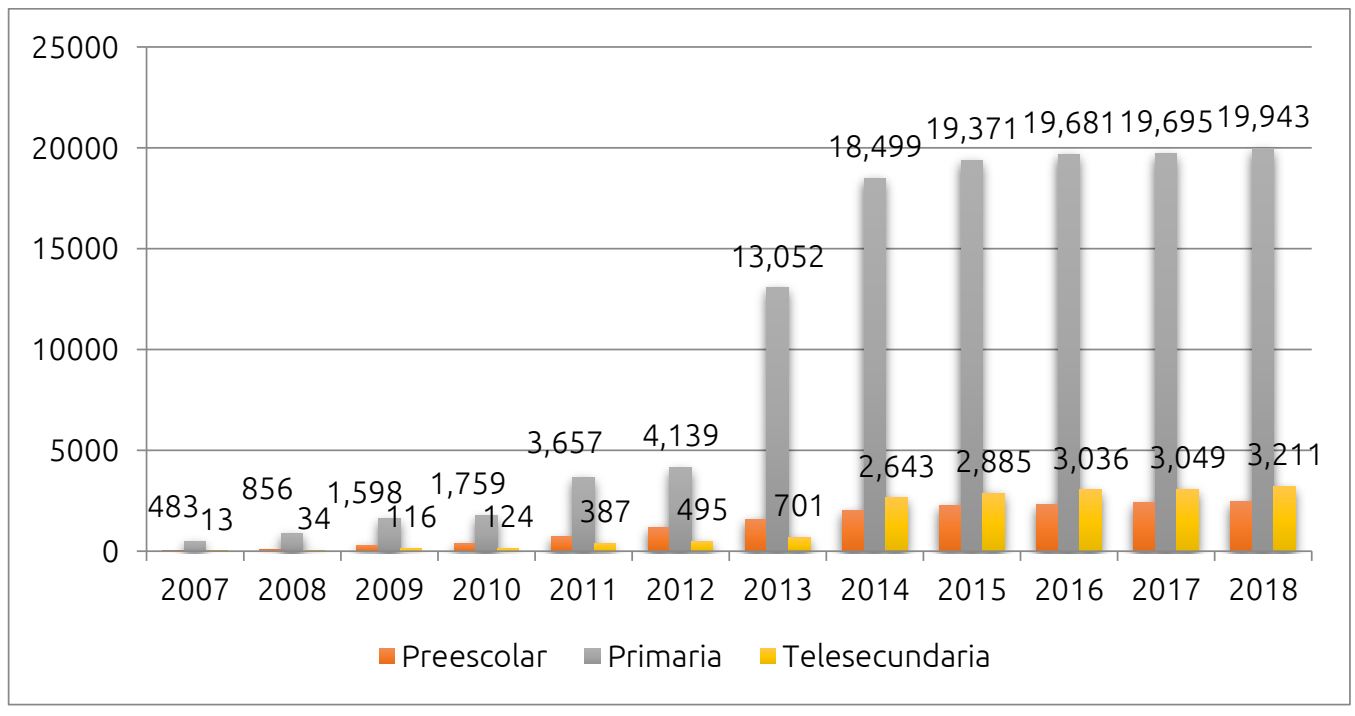

\section{Método}

El objetivo del estudio es hallar el efecto causal del PETC sobre el porcentaje de estudiantes que alcanzan cierto nivel de logro. Al respecto, el efecto tratamiento para una escuela $i$ es $Y_{i 1}-Y_{i 0}$ (donde $Y_{i 1}$ es el valor de la variable de resultado cuando la institución $i$ es beneficiaria del programa y $Y_{i 0}$ es el valor de la variable de resultado cuando el colegio $i$ no forma parte del programa).

Bajo este contexto no experimental, el efecto tratamiento de interés es el efecto tratamiento esperado sobre la población tratada (ATT), es decir:

$$
\begin{gathered}
A T T=E\left(Y_{i 1}-Y_{i 0} \mid T_{i}=1\right) \\
=E\left(Y_{i 1} \mid T_{i}=1\right)-E\left(Y_{i 0} \mid T_{i}=0\right)+E\left(Y_{i 0} \mid T_{i}=0\right)-E\left(Y_{i 0} \mid T_{i}=1\right) \\
=E\left(Y_{i 1} \mid T_{i}=1\right)-E\left(Y_{i 0} \mid T_{i}=0\right)+\gamma
\end{gathered}
$$

donde $T_{i}$ es una variable dicotómica que vale uno cuando la escuela $i$ es receptora del programa. El problema surge porque se puede observar $E\left(Y_{i 1} \mid T_{i}=1\right)$ pero no $E\left(Y_{i 0} \mid T_{i}=1\right)$, por lo que el estimador del efecto tratamiento tiene un sesgo $\gamma$. Si el sesgo es cero, el efecto promedio del programa es identificado. Sin embargo, el sesgo surge por la autoseleccción de las escuelas para participar en el programa. ${ }^{4}$ Además, una de las prioridades para incorporar una institución al programa es que cuente con rendimiento escolar bajo.

Si los padres están interesados en que sus hijos reciban mayor tiempo de instrucción escolar tienden a enviarlos a una escuela de tiempo completo; si esto que no es observable tiene impacto sobre el logro escolar de los niños, entonces la correlación entre el rendimiento escolar y las escuelas de tiempo completo puede ser atribuible a las características familiares. El sesgo también puede ser negativo, pues los niños con los resultados escolares más bajos pueden estar siendo canalizados a las escuelas de tiempo completo para reforzar su aprendizaje. ${ }^{5}$ Por consiguiente, dado que uno de los criterios para formar parte del PETC es que las escuelas tengan bajo rendimiento escolar, hay mayor probabilidad de que exista un sesgo

\footnotetext{
${ }^{4}$ La escuela se postula como candidata y cada entidad federativa selecciona, dentro de las postuladas, a las que formarán parte del programa.

${ }^{5}$ El motivo por el cual los niños tienen resultados educativos bajos puede ser diverso. La falta de interés por aprender o problemas de aprendizaje pueden ser dos causas que el programa por sí sólo no podría resolver.
} 
negativo en los resultados del programa.

Con la finalidad de corregir este sesgo, se asume que la diferencia entre las escuelas de los grupos de comparación es capturada por características observables. ${ }^{6}$ Bajo este supuesto, se hace uso del método de emparejamiento por puntaje de propensión para encontrar un estimador insesgado del efecto tratamiento. El método consiste en emparejar a los colegios que están en el programa con escuelas que cuentan con características observables similares pero que no formaron parte del programa.

Para hacer el emparejamiento, primero se emplea un modelo logístico para estimar la probabilidad de pertenencia al programa de cada colegio, esta probabilidad estimada es lo que se llama puntaje de propensión. Con base en ese puntaje se agrupa a las escuelas que pertenecen al programa y las que no, pero que cuentan con puntaje de propensión similar, para hacer comparación entre ellas. De acuerdo con Dehejia y Wahba (2002) el emparejamiento permite estimar el efecto tratamiento sobre la población tratada de la siguiente manera:

$$
\widehat{A T T}=\frac{1}{|N|} \sum_{i \in N}\left(Y_{i}-\sum_{j_{i} \in J_{i}} \omega_{i j} Y_{j}\right)
$$

donde $N$ es el conjunto de escuelas del grupo de tratamiento, $J_{i}$ es el conjunto de colegios emparejadas con la i-ésima escuela beneficiaria del programa, | $\mid$ indica la cardinalidad de un conjunto, $\omega_{i j}$ es el peso asociado a cada colegio $j$ emparejado con la escuela tratada $i$ y $Y$ es la variable de resultados.

Para formar los grupos de comparación con similar puntaje de propensión se hace uso del método de emparejamiento de radio, el cual utiliza como grupo de comparación a todas las escuelas cuyo puntaje de propensión esté dentro de un radio definido, llamado caliper. Una ventaja de este método es que permite el uso de unidades adicionales de comparación cuando los emparejamientos disponibles son buenos. Uno de los problemas es que, si el caliper es muy pequeño, algunas escuelas no tendrán su grupo de comparación, por lo que no podrán ser emparejadas y, por lo tanto, no pueden usarse para estimar el efecto. Aun así, mientras más pequeño es el caliper, la calidad de emparejamiento se mejora. Otro inconveniente de este método es que es difícil conocer a priori el nivel de tolerancia razonable para la selección del caliper. Con el objetivo de mejorar la calidad del emparejamiento, este método se combina emparejando colegios dentro de la misma entidad federativa y el efecto tratamiento es estimado sobre la población tratada dentro de estas entidades. En este caso, el efecto reportado es el promedio del ATT ponderado por el número de escuelas beneficiarias en el programa dentro de cada entidad.

Con fines de robustez, adicionalmente se realizan otros emparejamientos: radio con un caliper de 0.05, tres vecinos más cercanos y kernel. El método de tres vecinos más cercanos consiste en emparejar a cada institución tratada con las tres escuelas del grupo de control que se encuentran más cerca en términos del puntaje de propensión. El método de Kernel es un emparejamiento no paramétrico en el que se pondera inversamente a las escuelas del grupo de control según su distancia con cada escuela tratada.

\subsection{Datos}

Los datos utilizados para este estudio provienen de diversas fuentes de información. En esta sección se describe cada fuente y las variables que se utilizan de cada una de ellas.

La SEP reporta los resultados de las pruebas Planea a nivel escuela sobre el porcentaje de estudiantes que alcanzan cierto nivel de dominio de los aprendizajes claves del plan de estudios. Los niveles de logro son: insuficiente, apenas indispensable, satisfactorio y sobresaliente. El porcentaje de estudiantes con nivel de logro insuficiente y sobresaliente son las variables que se utilizan para medir el impacto del programa. Las asignaturas que se consideran en este examen son Matemáticas y Lenguaje y comunicación. De esta prueba

\footnotetext{
${ }^{6}$ Esto permite considerar la asignación del tratamiento como aleatoria y poder atribuir la diferencia entre las escuelas beneficiarias y no beneficiarias en la variable de resultado a la aplicación del tratamiento.
} 
se utilizan los resultados de los años 2015, 2017 y 2019.

Como uno de los criterios para ingresar al programa es contar con bajos niveles de logro educativo, se utiliza la calificación obtenida por las escuelas en la prueba ENLACE de 2013. Si bien es una prueba con baja confiabilidad, se asume que todas las telesecundarias cuentan con el mismo nivel de confianza en la prueba, de ahí que dicho puntaje sea un buen proxy del rendimiento académico de la escuela.

El cuestionario Formato 911 contiene información que la SEP solicita a cada escuela al inicio y al final de cada ciclo escolar. De este cuestionario se considera la información sobre el número de alumnos inscritos en la escuela, el número de docentes y el número de grupos. Se utilizan los datos del Formato 911 del ciclo escolar 2013-2014.

El Censo de Escuelas, Maestros y Alumnos de Educación Básica y Especial (CEMABE) (SEP, 2013) tuvo por objetivo conocer el estado de la educación en términos de infraestructura, mobiliario, servicios, composición de la plantilla docente, administrativa y estudiantil. Este censo tenía la finalidad de identificar las problemáticas del sistema educativo para ser atendidas de manera específica. La información de este censo permite restringir la muestra a las escuelas que en 2013 eran de un solo turno, que iniciaban clases entre 7 y 8 de la mañana y que no eran de jornada escolar completa. Además, de esta fuente de información se identifica el acceso a los servicios básicos de la escuela y la pertenencia a los programas de Escuelas de Calidad $^{7}$ y Fortalecimiento de la Educación Telesecundaria. ${ }^{8}$

El Censo de Población y Vivienda de 2010 permite utilizar información de la composición educativa de la población que reside en las localidades donde se ubican las escuelas. De igual forma, se hace uso del índice de rezago social de 2010 publicado por el Consejo Nacional de Evaluación de la Política de Desarrollo Social (CONEVAL) para controlar por las carencias que presentan las localidades. Del Decreto por el que se establece el Sistema Nacional para la Cruzada contra el Hambre (2013) se identifica a los municipios que forman parte de esta cruzada; y del Acuerdo donde se estipulan los lineamientos para el otorgamiento del apoyo a las entidades federativas para el Programa Nacional de Prevención del Delito (2018) se identifica a los municipios beneficiarios de este programa.

La muestra de análisis consiste en aquellas telesecundarias que fueron beneficiarias del PETC por primera vez en el ciclo escolar 2014-2015. Se consideran dos grupos de comparación: telesecundarias que no fueron beneficiarias del PETC en el ciclo escolar 2014-2015 y las telesecundarias que en los ciclos escolares 20152016, 2016-2017, 2017-2018 o 2018-2019 fueron incorporadas por primera vez como receptoras del programa. El primer grupo de comparación será llamado grupo de control de todas las telesecundarias, y el segundo será llamado grupo de control de escuelas que entraron después al programa. Estos grupos de comparación son restringidos a las entidades federativas en las que opera el programa. Además, se restringe la muestra a las escuelas con información válida en cada una de las fuentes de información. Así, el grupo tratamiento consta de 1248 observaciones. Mientras que el grupo de control de todas las telesecundarias consta de 8565 escuelas, y el grupo de comparación de escuelas que entraron después al programa es de 486.

En las figuras 2 a la 5 se presenta el porcentaje promedio de estudiantes con nivel de logro insuficiente y sobresaliente para el grupo tratamiento y para los grupos de comparación de todas las telesecundarias, al igual que para escuelas que entraron después. Se puede observar que en los colegios que fueron incorporadas al programa en 2014-2015, en los resultados de la prueba de 2015 tienen, en promedio, al $58.9 \%$ de sus estudiantes en el nivel de logro insuficiente en Matemáticas, y al $36.8 \%$ en Lenguaje y comunicación. En cambio, el grupo de control de todas las telesecundarias tiene, en promedio, al 56.7\% en Matemáticas y al 33\% en Lenguaje de estudiantes en el nivel de logro insuficiente. Es decir, en Matemáticas y Lenguaje hay, respectivamente, 2.2 y 3.8 puntos porcentuales más en el porcentaje de estudiantes con el nivel de logro más bajo en el grupo de escuelas pertenecientes al PETC que en el grupo de control. Estas

\footnotetext{
7 Su objetivo es mejorar el logro escolar mediante un cambio en la gestión educativa.

8 Busca mejorar el servicio de la educación telesecundaria mediante cursos propedéuticos, de reforzamiento y de regularización para incrementar el logro educativo de los alumnos.
} 
diferencias son significativas estadísticamente al 5\%. Entre estos grupos de comparación, para el año 2017 también se encuentra una brecha de 1.8 puntos porcentuales en Matemáticas y de 2.9 puntos porcentuales en Lenguaje. En contraste, en el año 2019 la diferencia sólo es significativa en la asignatura de lenguaje, siendo de 1.5 puntos porcentuales. La brecha en el porcentaje de estudiantes con nivel de logro insuficiente entre el grupo de tratamiento y el grupo de control de escuelas que entraron después no es estadísticamente significativa en ningún caso.

Por otra parte, en 2015 las escuelas del grupo de control de todas las telesecundarias tienen en promedio 0.98 y 0.6 puntos porcentuales más en Matemáticas y Lenguaje respectivamente, en el porcentaje de estudiantes con nivel de logro sobresaliente, que las escuelas beneficiarias del PETC. En 2017 la diferencia se amplía a 1.1 y 1.5 en Matemáticas y Lenguaje, respectivamente. Para el 2019 no hay diferencia estadísticamente significativa entre ambos grupos. La diferencia en el porcentaje de estudiantes con nivel de logro sobresaliente entre los grupos de tratamiento y el grupo de escuelas que entraron después al programa sólo es significativa al 5\% en el 2015; en este caso es 1.8 puntos porcentuales mayor el porcentaje de estudiantes con nivel de logro sobresaliente en las escuelas del grupo de control. De inicio parece que no hay beneficios en el logro educativo en las escuelas pertenecientes al PETC; de hecho, parece que muestran resultados educativos más bajos que en las escuelas de los grupos de comparación. Las figuras 2 , 3,4 y 5 muestran el porcentaje promedio de estudiantes en nivel de logro insuficiente y sobresaliente por grupo de comparación y por asignatura.

Figura 2. Porcentaje promedio de estudiantes en el nivel de logro Insuficiente en Matemáticas por grupo de comparación

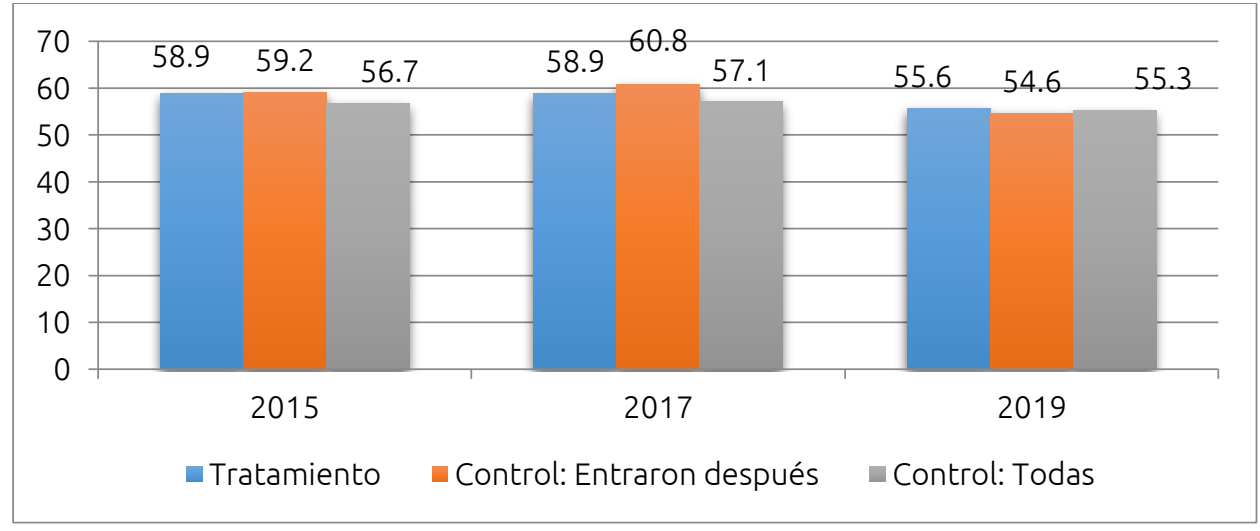

Figura 3. Porcentaje promedio de estudiantes en el nivel de logro Insuficiente en Lenguaje por grupo de comparación

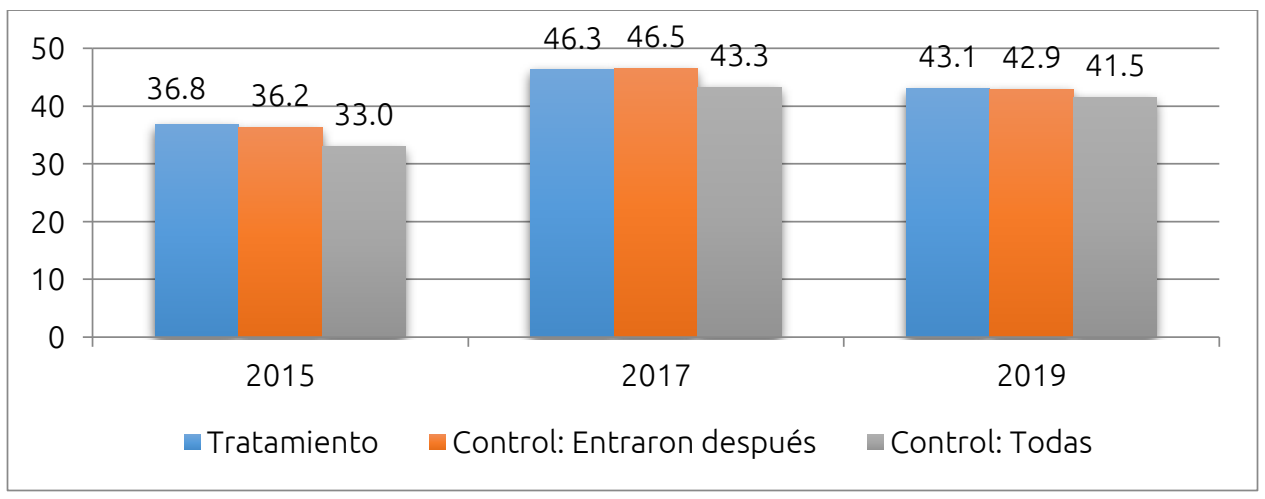


Figura 4. Porcentaje promedio de estudiantes en el nivel de logro Sobresaliente en Matemáticas por grupo de comparación

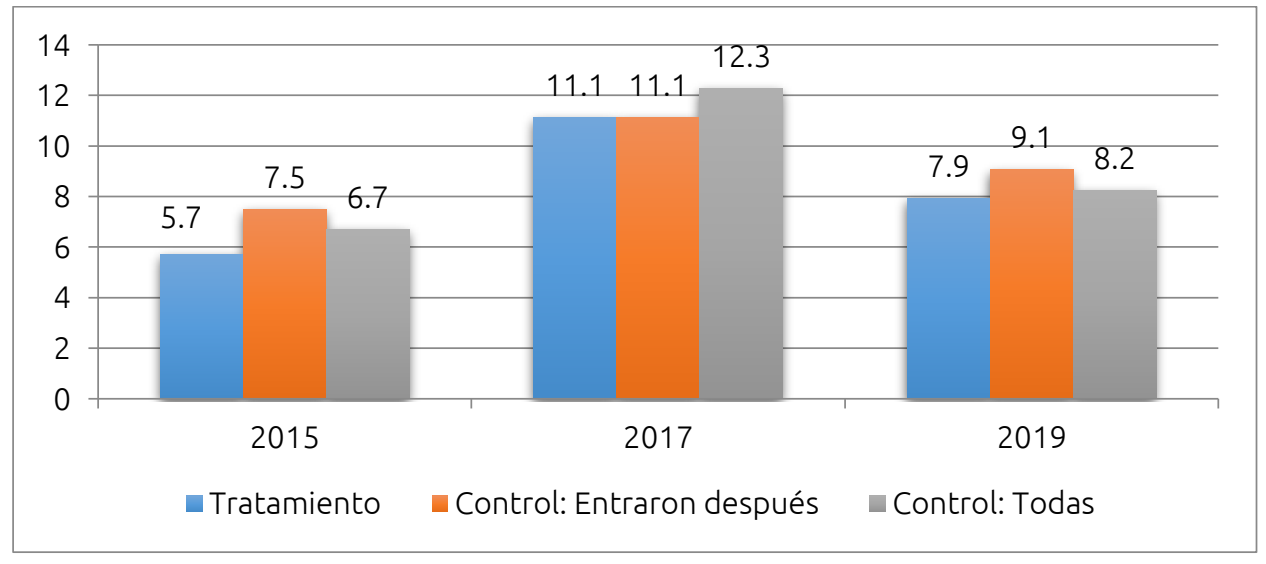

Figura 5. Porcentaje promedio de estudiantes en el nivel de logro Sobresaliente en Lenguaje por grupo de comparación

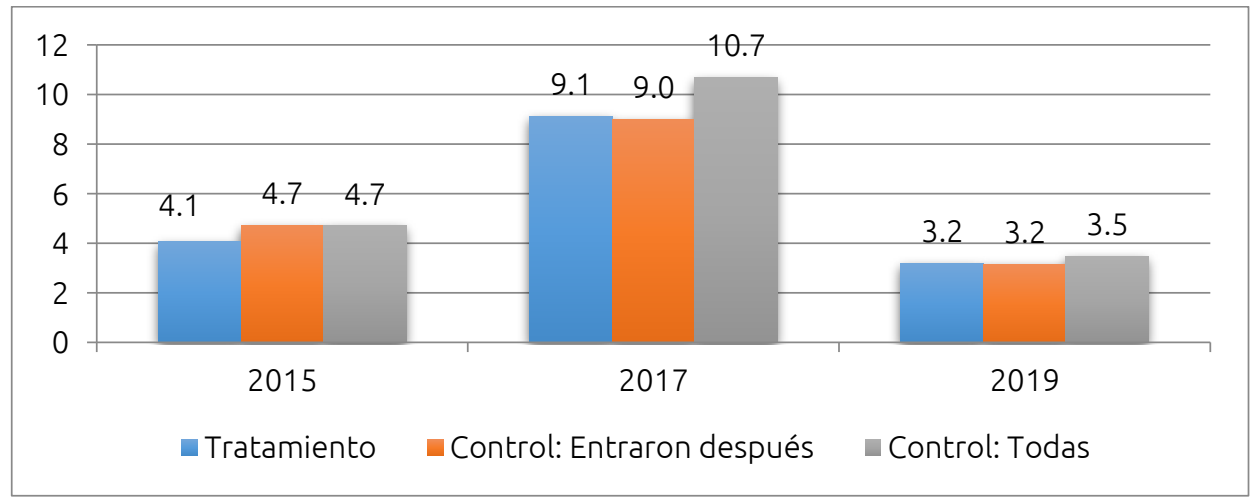

\section{Resultados}

De acuerdo con lo sugerido por Caliendo y Kopeinig (2008), para estimar el puntaje de propensión se emplean variables medidas antes del inicio del tratamiento, y se incluyen aquellas que afectan de forma simultánea tanto a la probabilidad de pertenecer al programa como al logro escolar. Explícitamente, las variables incorporadas en la estimación del puntaje de propensión son: las calificaciones en Español y Matemáticas en la ENLACE, el logaritmo del número de estudiantes inscritos, el número de alumnos por docente, el número de aulas, el número de hombres por mujeres, y variables indicadoras de que la escuela cuenta con sanitario, aulas de cómputo, talleres, piso de tierra y canchas deportivas, así como la pertenencia de la escuela a los programas de Escuelas de Calidad y al de Fortalecimiento de la Educación Telesecundaria. También se incluyen variables indicadoras de que la escuela no tiene servicio de agua y energía eléctrica. Además, se incorpora el porcentaje de personas de 15 años y más con educación básica incompleta, analfabeta, así como el porcentaje de personas entre 6 y 14 años que no asiste a la escuela, y el índice de rezago social; estas variables se refieren a las características de la localidad en la que se ubica la escuela. Se incluyen también variables indicadoras de que en el municipio donde se localiza el colegio opera la Cruzada contra el Hambre y el Programa Nacional para la Prevención Social de la Violencia y la Delincuencia.

Una vez estimado el puntaje de propensión usando cada grupo de comparación se hace el emparejamiento del grupo de tratamiento con los colegios del grupo de control cuyo puntaje se encuentra en un caliper de 0.05, comparando así escuelas dentro de la misma entidad federativa. Para fines de robustez se realizan los emparejamientos por medio de los tres vecinos más cercanos, emparejamiento Kernel y radio con caliper de 0.05. Los emparejamientos se realizaron usando el comando psmatch2 del programa estadístico STATA 
(Becker y Ichino, 2002).

Con estos tipos de emparejamiento se valida el supuesto de que la diferencia entre las escuelas de los grupos de compración es capturada por características observables. Para lo cual se emplea una diferencia de medias en las características observables, antes y después de hacer cada tipo de emparejamiento. Dado que las observaciones con similar puntaje de propensión deben tener la misma distribución de características observables independientemente del estatus de tratamiento; las unidades control y tratamiento deben ser en promedio observacionalmente idénticas después del emparejamiento. En este caso resulta que en menos del $10 \%$ de las variables observables se encuentran diferencias significativas entre los grupos de control y tratamiento. ${ }^{9}$

En la Tabla 1 se presenta el efecto promedio del PETC sobre el porcentaje de estudiantes con nivel de logro insuficiente y sobresaliente, con ambos grupos de comparación. Usando el grupo de control de todas las telesecundarias y con el emparejamiento de radio estratificado por entidad federativa se encuentra que en las escuelas del programa el porcentaje de estudiantes con nivel de logro insuficiente es menor en $0.99 \mathrm{y}$ 0.33 puntos porcentuales, en Matemáticas y Lenguaje respectivamente. En cuanto a las escuelas del PETC, el porcentaje de estudiantes con nivel de logro sobresaliente en Lenguaje es mayor en 0.25 puntos porcentuales y menor en Matemáticas en 0.72 puntos porcentuales. Resulta que ninguno de los efectos es estadísticamente significativo al 10\%. Lo mismo sucede con el grupo de control de escuelas que entraron después: no se observa algún efecto significativo en estas variables de interés.

Tabla 1. Efecto tratamiento promedio en los tratados sobre el porcentaje de estudiantes con nivel de logro insuficiente y sobresaliente. Por cada grupo de comparación y tipo de emparejamiento

\begin{tabular}{|c|c|c|c|c|}
\hline & \multicolumn{4}{|c|}{$\%$ Insuficiente } \\
\hline & \multicolumn{2}{|c|}{ Todas } & \multicolumn{2}{|c|}{ Entraron después } \\
\hline & Matemáticas & Lenguaje & Matemáticas & Lenguaje \\
\hline \multirow[t]{2}{*}{ R-Estr } & -0.99 & -0.33 & 1.41 & 0.59 \\
\hline & $(1.08)$ & $(0.95)$ & (1.53) & $(1.23)$ \\
\hline \multirow[t]{2}{*}{$R-0.05$} & $1.76^{* *}$ & $3.48 * * *$ & -0.39 & 0.79 \\
\hline & $(0.85)$ & $(0.67)$ & $(1.77)$ & $(1.32)$ \\
\hline \multirow[t]{2}{*}{$3 V M C$} & $2.00 *$ & $3.55^{* * *}$ & 1.46 & 2.61 \\
\hline & (1.18) & $(0.98)$ & $(2.36)$ & $(1.65)$ \\
\hline \multirow[t]{5}{*}{ Kernel } & $1.74 * *$ & $3.45 * * *$ & -0.38 & 0.83 \\
\hline & (0.80) & $(0.70)$ & $(1.82)$ & $(1.25)$ \\
\hline & \multicolumn{4}{|c|}{$\%$ Sobresaliente } \\
\hline & \multicolumn{2}{|c|}{ Todas } & \multicolumn{2}{|c|}{ Entraron después } \\
\hline & Matemáticas & Lenguaje & Matemáticas & Lenguaje \\
\hline \multirow[t]{2}{*}{ R-Estr } & -0.72 & 0.25 & -1.49 & 0.11 \\
\hline & $(0.88)$ & $(0.55)$ & $(1.28)$ & $(0.65)$ \\
\hline \multirow[t]{2}{*}{ R-0.05 } & $-0.82^{* *}$ & $-0.50 *$ & -1.33 & 0.10 \\
\hline & $(0.39)$ & $(0.26)$ & $(1.00)$ & $(0.50)$ \\
\hline \multirow[t]{2}{*}{$3 V M C$} & -0.96 & -0.39 & $-2.63 * *$ & -0.58 \\
\hline & $(0.62)$ & $(0.38)$ & $(1.23)$ & $(0.61)$ \\
\hline \multirow[t]{2}{*}{ Kernel } & $-0.81 * *$ & $-0.49 *$ & -1.37 & 0.09 \\
\hline & $(0.38)$ & $(0.29)$ & (1.03) & $(0.52)$ \\
\hline
\end{tabular}

Nota: R-Estr = método de emparejamiento utilizado para la estimación es el radio con un caliper de 0.05, con estratificación por entidad federativa. R-0.05 = método de emparejamiento utilizado para la estimación es el radio con un caliper 0.05. 3VMC = método de emparejamiento utilizado para la estimación es el de los tres vecinos más cercanos. Kernel = método de emparejamiento utilizado para la estimación es el Kernel con un ancho de banda de 0.06. Errores estándar entre paréntesis estimados mediante un remuestreo con 500 replicaciones. Efecto significativo al *10\%, **5\% y***1\%.

Con los otros tipos de emparejamiento, para el porcentaje de estudiantes en el nivel insuficiente se puede notar que con los dos grupos de comparación utilizados ninguno de estos efectos es estadísticamente significativo en Matemáticas. Para el caso de la materia de Lenguaje, con el grupo de comparación de todas

9 Otras medidas de calidad del emparejamiento se verificaron, tales como el sesgo estandarizado y pruebas de insignificancia conjunta de los regresores. En todos los casos las pruebas fueron satisfactorias. 
las telesecundarias se observa un efecto de signo positivo en el porcentaje de estudiantes con nivel de logro insuficiente, pero no es estadísticamente significativo. Con el grupo de comparación de escuelas telesecundarias el efecto es de aproximadamente 3.5 puntos porcentuales mayor en el porcentaje de estudiantes con nivel de logro insuficiente para las escuelas beneficiarias del PETC, relativo a las escuelas del grupo de control. Esto se observa en tres de los cuatro tipos de emparejamiento utilizados. En contraste, con el grupo de comparación de escuelas que entraron después al programa el efecto en esta variable no es estadísticamente significativo.

Los resultados con los otros emparejamientos respecto al porcentaje de estudiantes con nivel de sobresaliente son consistentes a los anteriormente mencionados. Es decir, no hay evidencia de algún efecto significativo del programa sobre el porcentaje de estudiantes con el nivel de desempeño más alto. Este resultado se cumple para ambas asignaturas.

No hay evidencia de efectos positivos del programa en el rendimiento escolar a un año de haber sido receptor. Con la finalidad de estudiar la evolución de los efectos encontrados, se hace uso de la muestra de escuelas que fueron por primera vez beneficiarias en el ciclo escolar 2014-2015. En las figuras 6 y 7, para cada campo disciplinar se presenta la evolución del efecto estimado del programa sobre el porcentaje de estudiantes con nivel de logro insuficiente y sobresaliente con el grupo de control de todas las telesecundarias. En la figura se muestra el efecto estimado usando el método de emparejamiento de radio con un caliper de 0.05 y estratificación por entidad federativa.

Figura 6. Evolución del efecto promedio en los tratados sobre el porcentaje promedio de estudiantes con nivel de logro Insuficiente

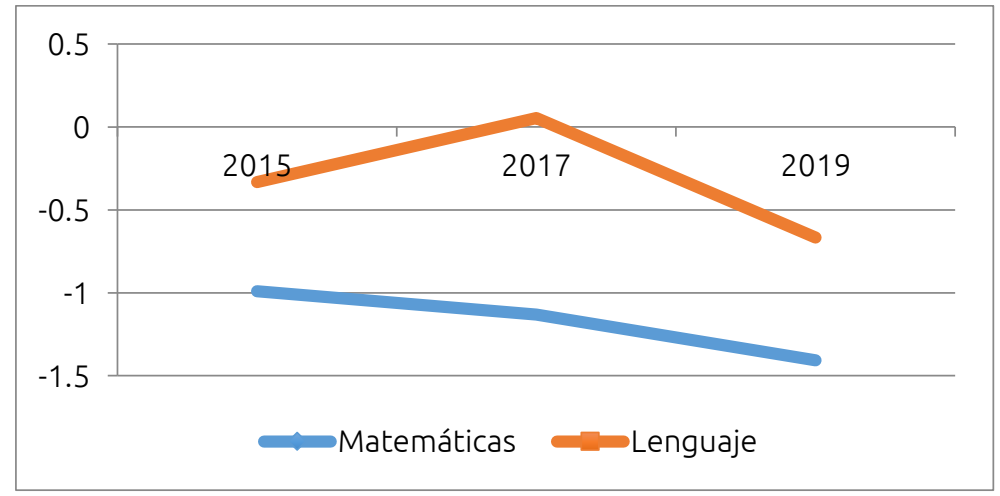

Figura 7. Evolución del efecto promedio en los tratados sobre el porcentaje promedio de estudiantes con nivel de logro Sobresaliente

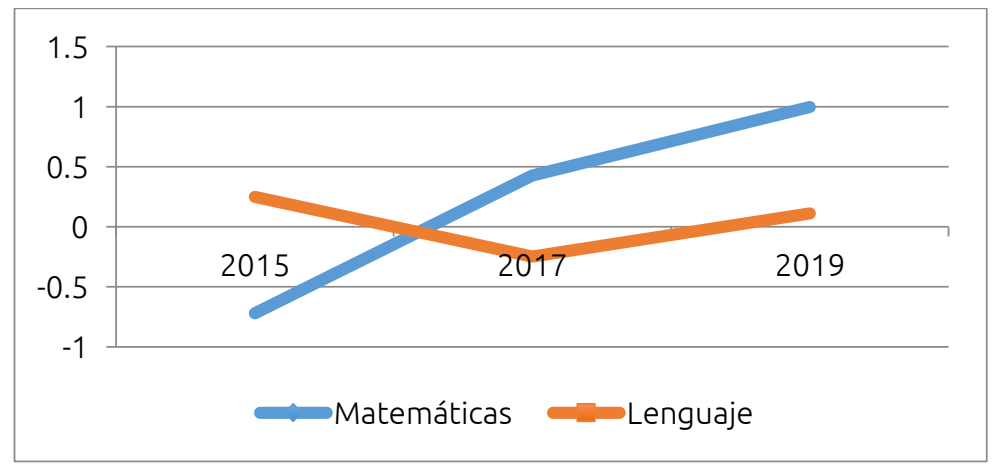

En 2015 el porcentaje de estudiantes con nivel de logro insuficiente muestra signo negativo en Matemáticas. Se puede notar que en 2017 el efecto del programa sobre el porcentaje de estudiantes con nivel de logro insuficiente es de -1.1 puntos porcentuales en Matemáticas, y para 2019 el efecto es de -1.4 
puntos porcentuales. Es decir, parece que el efecto del programa sobre el rendimiento en Matemáticas se va haciendo más grande conforme pasa el tiempo. A pesar de esto, el efecto del programa en ningún año es estadísticamente significativo. ${ }^{10}$ Lo mismo sucede con la asignatura de Lenguaje; los resultados se mantienen estadísticamente con el grupo de comparación de las escuelas que entraron después al programa (ver figura 8 y 9).

Figura 8. Evolución del efecto promedio en los tratados sobre el porcentaje promedio de estudiantes con nivel de logro Insuficiente

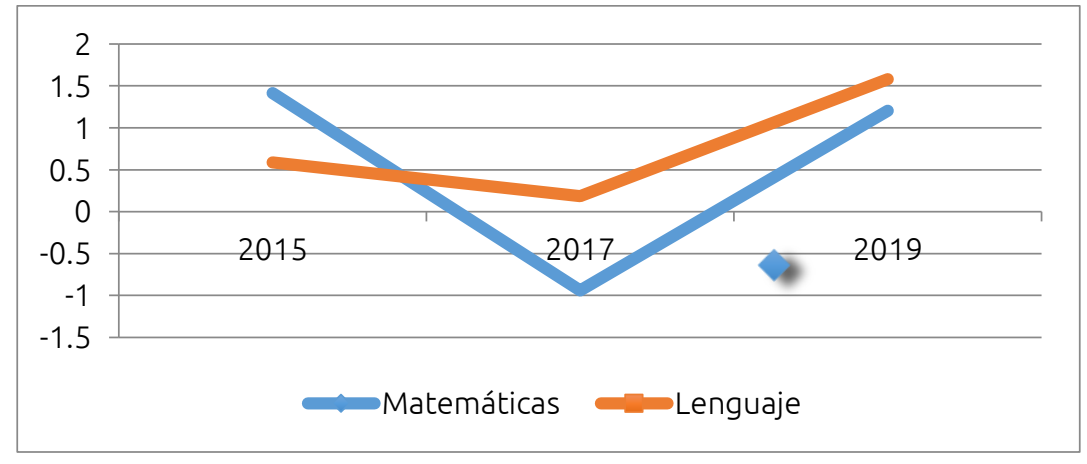

Figura 9. Evolución del efecto promedio en los tratados sobre el porcentaje promedio de estudiantes con nivel de logro Sobresaliente

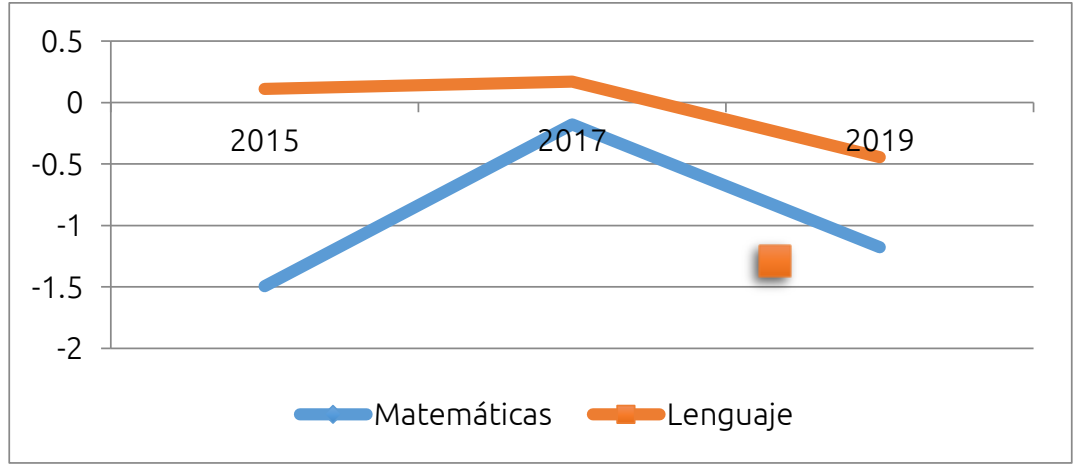

Respecto al efecto del programa sobre el porcentaje de estudiantes con nivel de logro sobresaliente, a través del tiempo se observa que presenta una tendencia creciente en la materia de Matemáticas, aun así este efecto no es estadísticamente significativo. Para la materia de Lenguaje tampoco hay efecto en 2017 y 2019. Estadísticamente, estos resultados se mantienen con todos los tipos de emparejamiento en la materia de matemáticas. Por el contrario, para la asignatura de Lenguaje sí hay efectos significativos en algunos casos. Sin embargo, al utilizar el grupo de comparación de telesecundarias que entraron después al programa no se observan efectos significativos en ningún año.

\section{Discusión y conclusiones}

En México existen al menos tres tesis de posgrado que evalúan el PETC (Andrade-Baena, 2014; CabreraHernández, 2015; Santillán, 2014). En los tres casos los autores utilizan los métodos de diferencias en diferencias y emparejamiento por puntajes de propensión. Con diversas muestras de escuelas y utilizando los resultados de la prueba ENLACE; los autores encuentran evidencia consistente de incrementos del logro escolar.

\footnotetext{
10 Aunque no se presentan en el presente documento, estos resultados se mantienen al usar los otros tipos de emparejamiento.
} 
Andrade-Baena (2014) presenta evidencia de que el PETC aumentó entre 0.06 y 0.13 desviaciones estándar los resultados en Matemáticas, y entre 0.07 y 0.13 desviaciones estándar los resultados de Español; asimismo, identifica que el programa tiene efectos acumulativos. Por su parte, Cabrera-Hernández (2015) encuentra magnitudes similares en los efectos, y presenta pruebas de que las escuelas con marginación alta exhiben los mayores efectos a los cuatro años de haber sido incorporadas al programa. Santillán (2014) halla que los mayores efectos del PETC se observan en los estudiantes del sexto grado, muestra que por un incremento de 10 días de clases hay un aumento de 1.9\% de una desviación estándar en Español, y conforme pasa el tiempo los efectos tienen incrementos marginales decrecientes.

La evaluación más reciente del programa la realizan Silveyra et al. (2018). Los autores usan un período de análisis de 2007 a 2016 y los datos de la ENLACE y la prueba Planea. Identifican que el PETC redujo en 4.6 y 1.7 puntos el porcentaje de estudiantes en el nivel de desempeño escolar más bajo en Matemáticas y Lenguaje, respectivamente; además, muestran que aumentó en 1.6 y 0.63 puntos porcentuales el porcentaje de estudiantes en el nivel de desempeño escolar más alto en Matemáticas y Lenguaje, respectivamente. También presentan evidencia de que los efectos se mantienen a lo largo del tiempo, y que son mayores para los alumnos de escuelas vulnerables y de alta marginalidad.

Cabe mencionar que las evaluaciones anteriores del PETC realizan el análisis del logro escolar para el nivel primaria, y en su mayoría hacen uso de los resultados de la ENLACE; de acuerdo con el INEE esta prueba censal presentó dificultades porque los resultados estaban asociados a incentivos económicos para los docentes, causando que los resultados fueran alterados y no hubiera confianza en las evaluaciones educativas. Es por eso que las autoridades educativas de México diseñaron la prueba Planea, que tiene la finalidad de dar a conocer el estado de la educación sin el objetivo de ordenar a las escuelas según su rendimiento, ni ofrecer incentivos asociados a los resultados de la prueba (INEE, 2018). Con esta prueba se implementan mecanismos que aseguran la confiabilidad de los resultados; de ahí que Planea sea más confiable que ENLACE.

A diferencia de los estudios previos sobre el programa, en este estudio se utilizan datos más veraces para medir el efecto causal del PETC sobre el nivel de logro escolar y se realiza un análisis de los efectos del programa en telesecundarias. Para cuantificar los efectos se usa la prueba Planea de los ciclos escolares 2014-2015, 2016-2017 y 2018-2019. Los resultados muestran evidencia de que el PETC no ha tenido efectos significativos en el nivel de logro de los estudiantes. En algunos casos, las escuelas del programa en la asignatura de Lenguaje presentan un aumento en el porcentaje de estudiantes con nivel de logro insuficiente relativo a las escuelas del grupo de control. El efecto es de 3.5 puntos porcentuales más, lo que equivale a un incremento del $10 \%$ en las escuelas tratadas, relativo al porcentaje de estudiantes en el nivel de logro insuficiente en las escuelas del grupo de control. Conforme pasa el tiempo tampoco se observa efecto significativo del programa en el nivel de logro de los estudiantes.

En una extensión del modelo de aprendizaje, Fisher et al. (1981) dividen al tiempo en tres clases: asignado, comprometido y de aprendizaje académico. El tiempo asignado es el análogo a la jornada escolar, el cual se considera en este trabajo. El tiempo comprometido es la parte del asignado en el que el estudiante pone atención, tratando de aprender o trabajando en alguna tarea específica. El tiempo de aprendizaje académico es una parte del tiempo comprometido en el que el estudiante experimenta un aprendizaje significativo. Una conclusión de este modelo es que los estudiantes aprenden más cuanto mayor es el tiempo comprometido; y el aprendizaje se maximiza conforme se incrementa el tiempo de aprendizaje significativo.

Como el PETC otorga la libertad a las escuelas de destinar el tiempo asignado a lo que consideran conveniente, en este sentido los efectos nulos observados para el nivel secundaria pueden ser debido a una falla en la organización que ha limitado el incremento en el tiempo comprometido y el tiempo de aprendizaje significativo. La falta de actividades didácticas que estimulen la participación activa de los estudiantes, la infraestructura escolar inadecuada y el poco involucramiento de los padres de familia en las actividades académicas, son problemas que han sido detectados en el programa y que afectan su eficiencia (SEP, 2017). 
Para el programa chileno Jornada Escolar Completa, Martinic y Villalta (2015) encuentran evidencia de que a medida que pasa el tiempo, los docentes del programa mejoran la organización de sus clases, exponen más los contenidos y destinan más tiempo para dar instrucciones sobre los procedimientos, todo esto mejora el aprendizaje de los estudiantes. Por lo que podría esperarse que conforme siga avanzando el tiempo se observen resultados positivos del programa en el nivel de secundaria.

Otro aspecto relevante que debe tomarse en cuenta es que la escuela telesecundaria es una modalidad educativa en la que un único profesor es encargado de la formación integral de los estudiantes y su principal apoyo son cápsulas de televisión. Este tipo de escuelas están caracterizadas por la carencia de infraestructura escolar, contar con materiales de trabajo inapropiados y la falta de capacitación al personal (Ramírez, 2019). Sin duda, estas condiciones atenúan los efectos que el programa podría alcanzar.

Otro aspecto relevante que debe tomarse en cuenta es que los subsistemas educativos contienen elementos propios que pueden atenuar los efectos postivos del programa; por ejemplo, por mencionar algunos, carencia de infraestructura adecuada y falta de materiales didacticos actualizados. Cabe recordar que la escuela telesecundaria es una modalidad educativa en la que un único profesor es encargado de la formación integral de los estudiantes y su principal apoyo son cápsulas de televisión.

A pesar de que actualmente no se observan efectos en el aprendizaje en secundaria; los efectos del PETC en la deserción, la delincuencia y el embarazo adolescente son desconocidos, por lo que se requiere indagar sobre estos aspectos en futuras investigaciones.

\section{Referencias}

Abdullah, A., Doucouliagos, H. y Manning, E. (2015). Does education reduce income inequality? A metaregression analysis. Journal of Economic Surveys, 29(2), 301-316. https://doi.org/10.1111/joes.12056

Acuerdo número 704 por el que se emiten las Reglas de Operación del Programa Escuelas de Tiempo Completo, Diario Oficial de la Federación [D.O.F.], 28 de diciembre de 2013 (México).

Acuerdo por el que se establecen los lineamientos para el otorgamiento de apoyos a las entidades federativas en el marco del programa nacional de prevención del delito, Diario Oficial de la Federación [D.O.F.], 24 de enero de 2018 (México).

Andrade-Baena, G. (2014). Improving academic achievement through extended school-days: Evidence from escuelas de tiempo completo in Mexico (Tesis doctoral no publicada). Stanford University.

Becker, S. e Ichino, A. (2002). Estimation of average treatment effects based on propensity scores. The Stata Journal, 2(4), 358-377. https://doi.org/10.1177/1536867X0200200403

Bellei, C. (2009). Does lengthening the school day increase students' academic achievement? Results from a natural experiment in Chile. Economics of Education Review, 28(5), 629-640.

https://doi.org/10.1016/j.econedurev.2009.01.008

Bloom, B. (1973). Recent developments in mastery learning. Educational Psychologist, 10(2), 53-57. https://doi.org/10.1080/00461527309529091

Cabrera-Hernández, F. (2015). Does lengthening the school day increase students' academic achievement? Evidence from a natural experiment (Tesis Doctoral no publicada). University of Sussex Business School.

Caliendo, M. y Kopeinig, S. (2008). Some practical guidance for the implementation of propensity score matching. Journal of Economic Surveys, 22(1), 31-72. https://doi.org/10.1111/j.1467-6419.2007.00527.x

Card, D. (1999). The causal effect of education on earnings. En O. Ashenfelter y D. Card, Handbook of labor economics (Vol. 3) (pp. 1801-1863). Elsevier. 
Carroll, J. (1963). A model of school learning. Teachers College Record, 64(8), 723-733.

Cattaneo, M. A., Oggenfuss, C. y Wolter, S. (2017). The more, the better? the impact of instructional time on student performance. Education Economics, 25(5), 433-445.

https://doi.org/10.1080/09645292.2017.1315055

Declaración de Cochabamba (2001). Recomendaciones sobre Políticas educativas al inicio del siglo XXI. Séptima reunión del Comité Regional Intergubernamental del Proyecto de Educación en América Latina y el Caribe (PROMEDLAC VII)

Decreto por el que se establece el Sistema Nacional para la Cruzada contra el Hambre, Diario Oficial de la Federación [D.O.F.], 22 de enero de 2013 (México).

Decreto por el que se reforma el artículo 3o., en sus fracciones III, VII y VIII, Diario Oficial de la Federación [D.O.F.], 26 de febrero de 2013 (México).

Dehejia, R. y Wahba, S. (2002). Propensity score-matching methods for nonexperimental causal studies. Review of Economics and Statistics, 84(1), 151-161. https://doi.org/10.1162/003465302317331982

Fisher, C., Berliner, C., Filby, N., Marliave, R., Cahen, S. y Dishaw, M. (1981). Teaching behaviors, academic learning time, and student achievement: An overview. The Journal of Classroom Interaction, 17(1), 2-15. https://www.jstor.org/stable/43997772

Hanushek, E. y Wößmann, L. (2007). The role of education quality for economic growth. The World Bank.

Instituto Nacional para la Evaluación de la Educación (INEE). (2018). Plan nacional para la evaluación de los aprendizajes (Planea). Documentos rectores. México.

https://www.inee.edu.mx/evaluaciones/planea/planea-documento-rector/

López-Aguilar, M. (2013). Una reforma educativa contra los maestros y el derecho a la educación. El Cotidiano, (179), 55-76. https://issuu.com/elcotidiano/docs/179

Martinic, S. y Villalta, M. (2015). La gestión del tiempo en la sala de clases y los rendimientos escolares en escuelas con jornada completa en chile. Perfiles Educativos, 37(147), 28-49.

https://doi.org/10.22201/iisue.24486167e.2015.147.47260

Meroni, E. y Abbiati, G. (2016). How do students react to longer instruction time? Evidence from Italy. Education Economics, 24(6), 592-611. https://doi.org/10.1080/09645292.2015.1122742

OCDE. (2016a). Pisa 2015 resultados clave. Autor. https://www.oecd.org/pisa/pisa-2015-results-in-focusESP.pdf

OCDE (2016b). Programa para la evaluación internacional de alumnos (PISA). PISA 2015-resultados. http://www.oecd.org/pisa/PISA-2015-Mexico-ESP.pdf

Ramírez, J. (2019). ¿Las políticas educativas mexicanas reforman o deforman la educación en las telesecundarias? Desafíos de la escuela telesecundaria, desde la perspectiva docente. UPN, unidad 241.

Rivkin, S. y Schiman, J. C. (2015). Instruction time, classroom quality, and academic achievement. The Economic Journal, 125(588), F425-F448. https://doi.org/10.1111/ecoj.12315

Santillán, A. (2014). Ensayos sobre educación y el mercado laboral en México (Tesis doctoral no publicada). El Colegio de México. 
Secretaría de Educación Pública (2013). Censo de Escuelas, Maestros y Alumnos de Educación Básica y Especial. http://www.censo.sep.gob.mx/

Secretaría de Educación Pública (2017). Diagnóstico Actualizado. Programa Escuelas de Tiempo Completo. https://www.gob.mx/cms/uploads/attachment/file/261716/S221 Programa Escuelas de Tiempo Comp leto - Diagno stico 2017.pdf

Silveyra, M., Yañez, M. y Bedoya, J. (2018). ¿Qué impacto tiene el programa de escuelas de tiempo completo en los estudiantes de educación básica?: Evaluación del programa en México 2007-2016. Banco Mundial. http://documents.worldbank.org/curated/en/157301536217801694/pdf/129769-WP-PUBLIC-SPANISHEscuelasTiempoCompletoBajaRes.pdf 